Décadrages Décadrages

cınéma, à travers champs Cinéma, à travers champs

39 | 2018

Jeu vidéo et cinéma

\title{
Pour une analyse des discours sur le jeu vidéo : l'exemple des « cinématiques »
}

Selim Krichane et Yannick Rochat

\section{(2) OpenEdition}

1 Journals

Édition électronique

URL : https://journals.openedition.org/decadrages/1341

DOI : $10.4000 /$ decadrages.1341

ISSN : 2297-5977

Éditeur

Association Décadrages

Édition imprimée

Date de publication : 1 décembre 2018

Pagination : 62-92

ISBN : 978-2-9700963-5-1

ISSN : 2235-7823

Référence électronique

Selim Krichane et Yannick Rochat, « Pour une analyse des discours sur le jeu vidéo : l'exemple des " cinématiques » », Décadrages [En ligne], 39 | 2018, mis en ligne le 05 février 2021, consulté le 04 avril 2022. URL : http://journals.openedition.org/decadrages/1341 ; DOI : https://doi.org/10.4000/ decadrages. 1341

(B) Décadrages 


\section{Pour une analyse des discours sur le jeu vidéo: l'exemple des "cinématiques »}

LE JEU vidÉo God of War (Santa Monica Studio), paru le 20 avril 20I8, a été qualifié à l'unanimité de jeu en «plan-séquence» par la presse spécialisée, nous rappelant ainsi la prégnance du lexique cinématographique au sein des schèmes de perception et d'évaluation des productions vidéoludiques. L'absence de «saute» dans le flux visuel du jeu amène un journaliste de Libération à le qualifier de "plan-séquence de 30 heures » ${ }^{1}$, avant de se fendre d'une référence à La Corde (Alfred Hitchcock, I948, E-U), puis d'ajouter: «Pas un temps de chargement, pas un plan de coupe, pas un fondu au noir ne vient interrompre la progression d'une forêt enneigée à la cime d'une montagne. Les cinématiques n’ont pas disparu mais la transition reste quasi invisible. ${ }^{2}$

Cet exemple illustre la place de choix qu'occupe le cinéma en qualité de «médiateur des imaginaires ${ }^{3}$ dans le contexte de l'industrie du jeu vidéo, marquée par des logiques généralisées d'hybridation et de convergence médiatiques et par une histoire culturelle riche en synergies industrielles et économiques. Il témoigne également du fait que parallèlement aux termes empruntés au cinéma, le lexique vidéoludique a aussi créé ses propres notions, parfois au contact d'autres médias, comme en atteste le vocable «cinématique» ${ }^{4}$ (ou «cut-scene» en anglais, suivant la terminologie majoritairement employée).

Dans le cadre de cet article, nous proposons de retracer l'histoire de la notion de «cinématique» à partir d'un corpus de magazines spécialisés publiés entre I982 et I998. Afin de conduire une «lecture distante» de ce corpus textuel composé de 70 ooo pages environ, nous avons développé une méthodologie d'analyse des données utilisant des outils informatiques inédits dans le domaine des game studies. L'objectif de cette recherche consiste à proposer une alternative à l'étude des relations entre le cinéma et les jeux vidéo (fondée sur l'analyse d'éléments formels) au profit de l'analyse des discours dans une perspective résolument
1 Marius Chapuis, «God of War: hache de raison", Libération, 27 avril 2018, version en ligne: http://next.liberation.fr/ images/2018/04/27/god-of-war-hachede-raison_ı646376.

2 Id.

3 Alexis Blanchet, Des pixels à Hollywood. Cinéma et jeu vidéo, une histoire économique et culturelle, Houdan, Pix'n Love, 20Io, p. 234.

4 Notons que dans le domaine du cinéma, le terme de «cinématique» («cinematic» en anglais) constitue une notion théorique qui apparaît dans les travaux d'Eisenstein et qui permet à l'auteur de reconstituer une généalogie des dispositifs cinématographiques à travers d'autres champs comme la poésie, la littérature ou encore la peinture. Voir à ce titre Sergueï Mikhaïlovitch Eisenstein, Cinématisme - Peinture et cinéma, Dijon, Les Presses du réel, 2009 [I980]. Le terme a récemment été repris par certains théoriciens du cinéma élargi, comme Pavle Levi (Cinema by Other Means, New York/Oxford, Oxford University Press, 2012). 
5 Bernard Perron, Hugo Montembeault, Andréane Morin-Simard et Carl Therrien, «The Discourse Community's Cut: Video Games and the Notion of Montage», dans Jeff Thoss et Michael Fuchs (éd.), Intermedia Games - Games Inter Media, Boolmsbury, à paraître en 2018.

6 Cette appréhension, plus méfiante, apparaît notamment chez Mathieu Triclot, Philosophie des jeux vidéo, Paris, La Découverte, 20II.

7 Espen Aarseth, Cybertext: Perspective on Ergodic Literature, Baltimore, Johns Hopkins University Press, I997, pp. 2-13;59-6o.

8 Markku Eskelinen, "The Gaming Situation», Game Studies, vol. I, $\mathrm{n}^{\circ} \mathrm{I}$, 200I, p. 2.

9 Gonzalo Frasca, «Ludology Meets Narratology: Similitudes and Differences between (Video) Games and Narratives». Disponible en ligne: www. ludology.org/articles/ludology.html. historique. Cette recherche vise à élucider l'émergence et la naturalisation d'une notion clé au sein des discours sur le jeu vidéo, en retraçant les mécanismes qui régissent l'évolution des termes employés pour qualifier le jeu vidéo.

\section{Cinéma et jeu vidéo}

Le domaine des game studies, qui s'est institutionnalisé à partir de la fin des années I990, regroupe un faisceau pluriel de recherches s’inscrivant dans des disciplines et des méthodologies variées, réunissant ainsi, autour d'un objet commun, des approches redevables aux sciences humaines et sociales, aux sciences de l'ingénieur, autant qu'aux réflexions théoriques issues du domaine du game design ou encore des études numériques. En considérant la part des travaux qui provient des sciences humaines, on remarque que la référence au «cinéma» a joué un rôle déterminant dans l'élaboration d'un cadre théorique dès la fin des années i990. A ce titre, on peut noter à la suite de Bernard Perron et de ses collègues de l'Université de Montréal que le cinéma a souvent constitué un objet de controverse dans le contexte des game studies, pouvant être considéré par certains comme un bon objet de comparaison - notamment dans le cadre des recherches en intermédialité - ou, au contraire, désigné en tant que figure repoussoir ${ }^{5}$, dont la proximité en termes de représentations serait trompeuse et masquerait, en définitive, les spécificités du jeu vidéo ${ }^{6}$.

La place de choix occupée par le cinéma - alors même que les études cinématographiques, en tant que modèle théorique et méthodologique, sont relativement peu mobilisées - peut s'expliquer par trois facteurs distincts.

En premier lieu, cette comparaison médiatique aura été renforcée par un fait propre à l'histoire institutionnelle des game studies et à leur consolidation: celle-ci s'est faite en partie sous l'égide de chercheurs provenant initialement de l'étude des médias et dont le capital de recherche s'était constitué ailleurs, à partir d'autres objets d'études.

En deuxième lieu, on peut noter que l'émergence des game studies aura été marquée par un vif débat théorique (autour de la pertinence des approches narratologiques appliquées au jeu vidéo) qui a joué une fonction primordiale dans la constitution du «champ» (au sens institutionnel et sociologique). De nombreux chercheurs, à l'instar d'Espen Aarseth ${ }^{7}$, Markku Eskelinen ${ }^{8}$ ou Gonzalo Frasca ${ }^{9}$, ont alors défini leur approche 
en opposition aux études littéraires et cinématographiques dans un effort de définition des spécificités de ce nouveau média, et par peur de voir son étude phagocytée par des disciplines lui étant «étrangères ». D'autres chercheurs ont au contraire perçu le cinéma comme une ressource potentielle pour l'étude du jeu vidéo. C’est par exemple le cas de Janet Murray qui proposait à la fin des années I990 de mesurer les qualités narratives et immersives du jeu vidéo à l'aune du cinéma ${ }^{10}$.

En troisième lieu, le foisonnement d'études comparatives - auquel participe ce numéro de Décadrages! - aura certainement été favorisé par la dimension éminemment audiovisuelle du jeu vidéo, qui s’inscrit dans la généalogie des médias d'audiovision et dont les conditions de possibilités historiques recoupent bien souvent d'autres séries médiatiques, comme le cinéma, ou encore la télévision" ${ }^{11}$. Afin d'accommoder une place à la dimension audiovisuelle dans l'étude du jeu vidéo, sans pour autant négliger son caractère ludique et simulationnel, Frans Mäyrä parle de «double structure du jeu vidéo». Le chercheur finlandais indique alors qu'«en tant que simulations ludiques combinées à un médium audiovisuel et numérique, les jeux numériques [digital games] offrent aux joueurs à la fois l'accès à une 〈enveloppe〉 [shell] (couches représentationnelles) mais également à un 〈noyau〉 [core] (le gameplay)»12.

Une tendance importante des travaux qui s'attèlent à penser le jeu vidéo à l'aune du cinéma (et réciproquement) consiste à chercher des points de jonction formels, à partir d'un corpus restreint de jeux et de films, afin de cartographier les «similitudes» entre ces deux séries médiatiques. Les logiques d'identification du joueur à son avatar, les dimensions narratives et fictionnelles du jeu vidéo, les processus de remédiatisation ou encore les synergies industrielles entre ces deux industries culturelles ont ainsi fait l'objet d'une littérature secondaire abondante durant ces vingt dernières années ${ }^{13}$.

10 Voir Janet Murray, Hamlet on the Holodeck. The Future of Narrative in Cyberspace, MIT Press, Cambridge, I998 [I997], p. 2. Pour Murray, à l’orée du $\mathrm{XxI}^{\mathrm{e}}$ siècle, «l'ordinateur ressemble de plus en plus à la caméra cinématogra- phique des années i89o: une invention révolutionnaire que les humains sont sur le point d'utiliser en guise de conteur envoûtant».

11 Les consoles de salon, qui apparaissent au début des années I970 et qui constituent encore aujourd'hui un mode de consommation majeur du jeu vidéo, dépendent très directement d'une infrastructure télévisuelle pour fonctionner. Dans son étude du dispositif technique de Pong (Atari, I972), Henry Lowood a ainsi montré très judicieusement que la console constituait davantage un aménagement de la machinerie télévisuelle qu'une quelconque forme de machine informatique. Comme le note Lowood, «le jeu originel n'exécutait pas une seule ligne de code informatique. Il n'utilisait pas de microprocesseur, ni de circuit intégré personnalisé». Il en va de même pour le créateur de la première console de salon commercialisée en 1972, Ralph E. Baer, ingénieur électrique travaillant dans le domaine de la télévision (à l’image d'Al Alcorn, ingénieur en chef dans la création de Pong) dont l'effort de création d'une console de «jeu TV»s'inscrit dans la lignée des dispositifs télévisuels et non pas informatiques. Henry Lowood, "Videogames in Computer Space: The Complex History of Pong», IEEE Annals of the History of Computing, IEEE Computer Society, 2009, p. I2.

12 Frans Mäyrä, "Getting into the Game: Doing Multidisciplinary Game Studies", dans Mark J.P. Wolf et Bernard Perron (éd.), The Video Game Theory Reader 2, New York, Routledge, 2009, p.317. Voir également Frans Mäyrä, Introduction to Game Studies: Games in Culture, Londres/New York, Sage Publications, 2008 , pp. I7-I8.

13 Ce recensement apparaît notamment dans l'introduction à l'ouvrage collectif dirigé par Geoff King et Tanya Krzywinska, ScreenPlay: cinema/videogames/interfaces, Londres/New York, Wallflower Press, pp. I-32. 
14 La classification de Sandy Baczowski est particulièrement étendue et englobe notamment le «temps», l'«espace», le «montage» ou encore le «cadrage» dans sa liste des éléments partagés. Sandy Baczowski, La Contamination du cinéma américain contemporain par les jeux vidéo: convergences et divergences, thèse de doctorat, Université de Toulouse II - Le Mirail, p. 48, 2005. Martin Picard tire profit du travail de Baczowski dans sa propre thèse et lui emprunte notamment ses catégories de "remédiatisation» [remediation]. Martin Picard, Pour une esthétique du cinéma transludique: Figures du jeu vidéo et de l'animation dans le cinéma d'effets visuels du tournant $d u X X I^{e}$ siècle, thèse de doctorat sous la direction de Livia Monnet et Bernard Perron, Université de Montréal, 2009 (non publiée). Disponible en ligne: www.ludov.ca.

15 Federico Giordano, Ivan Girina et Riccardo Fassone (éd.), Game: The Italian Journal of Game Studies, $\mathrm{n}^{\circ}$ 4, dossier «Re-framing video games in the light of cinema», 20I5, Disponible en ligne: www. gamejournal.it/intro_5/.

16 Mark J. P. Wolf, «Inventing Space. Towards a Taxinomy of On- and OffScreen Space in Video Games», Film Quarterly, n 5I, I997, pp. II-23. L'article a été réédité dans Mark J. P. Wolf, The Medium of the Video Game, Austin, University of Texas Press, 200I, pp. 77-89.

17 Elsa Boyer et al. (éd.), Voir les jeux vidéo. Perception, construction, fiction, Montrouge, Bayard Editions, 2012, p. 7.

18 Bernard Perron et Carl Therrien, «Da Spacewar! a Gears of War, o comme l'immagine videoludica è devintata più cinematografica», bianco e nero, $n^{\circ} 564$, mai-août 2009. Disponible en français en ligne: www.ludov.ca.
Il en va ainsi du travail de Sandy Baczowski qui tente d'établir un répertoire des «points communs» entre cinéma et jeu vidéo afin de repérer l'ensemble des procédés de «remédiatisation» qui affectent, dans le contexte contemporain, les images du jeu vidéo et celles du cinéma ${ }^{14}$. On peut également citer le travail plus récent des chercheurs italiens Federico Giordano, Ivan Girina et Riccardo Fassone qui proposaient en 2015 d'«envisager le jeu vidéo à la lumière du cinéma» afin de faire retour sur les «relations complexes entre le cinéma et les jeux vidéo » ${ }^{15}$, tout en indiquant que celles-ci ont fait l'objet de nombreuses controverses. Les auteurs italiens nous rappellent que ces relations ont été thématisées très tôt, notamment par Mark J. P. Wolf dans sa typologie des espaces du jeu vidéo, qui, dès I997, puisait dans les ressources offertes par l'analyse des espaces cinématographiques (principalement à travers la typologie du hors-champ établie par Noël Burch) ${ }^{16}$.

Parmi les travaux qui proposent d'«interroger le jeu vidéo depuis ses images ${ }^{\mathbf{1 7}}$, on peut également retenir l'article de Bernard Perron et Carl Therrien qui isole trois «éléments formels» afin d'expliquer la «cinématographicité» croissante des images du jeu vidéo. Aux côtés de l'utilisation d'une «caméra virtuelle» et de la «qualité mimétique de l'image vidéoludique», les auteurs retiennent l'utilisation croissante de séquences non interactives à partir des années $1990^{18}$ - séquences qui sont, à partir de cette décennie, qualifiées de «cinématiques».

\section{Les « cinématiques » : facteur de convergence}

Les «cinématiques» sont très souvent désignées comme un facteur décisif d’intermédialité au sein des travaux tentant d'«explorer le territoire qui mène de la maison cinéma au terrain vague du jeu vidéo » ${ }^{19}$. Alexis Blanchet propose de définir la cinématique comme «un segment visuel de l'œuvre vidéoludique qui privilégie momentanément la posture de spectateur à celle de joueur», avant d'en proposer une typologie détaillée. Celle-ci mettrait en pause l'activité kinesthésique du joueur au profit d'une position de spectateur, la cinématique ayant souvent été traitée en regard de la question de l'interactivité du médium vidéo-

19 Paul Sztulman, «Les explorateurs des abîmes", dans Elsa Boyer et al. (éd.), Voir les jeux vidéo, op. cit., p. 4I. 
ludique. Aussi James Newman peut-il esquisser un cadre d'analyse graduel de l'interactivité vidéoludique à partir de ces «séquences filmiques non interactives ${ }^{20}$. Alors qu'il prenait la «défense» des cinématiques en 2002, Rune Klevjer s'opposait à l'idée selon laquelle leur non-interactivité les excluait du jeu, en suggérant que les cinématiques colorent nécessairement l'activité de jeu qu'elles encadrent, tout en offrant une ressource narrative considérable au médium ${ }^{21}$. Interactivité et narration auront ainsi constitué des thématiques centrales dans les textes académiques consacrés aux cinématiques - en sus de la question de l'intermédialité lorsqu'elle est posée frontalement-, nous rappelant que les recherches s'y affairant privilégient souvent les questions théoriques à l'étude historique de cette «figure» emblématique du jeu vidéo de grande consommation ${ }^{22}$.

Parmi les travaux qui proposent d'offrir un cadre historique à l'étude des «cinématiques», plusieurs chercheurs remarquent que la généralisation de cette figure, qui attire aussi bien l'attention des joueurs, des critiques et des chercheurs dès les années I990, s’inscrit par ailleurs dans une histoire plus longue des séquences non interactives du jeu vidéo. Les exemples canoniques des années I990, à l’image des cinématiques de Final Fantasy VII (Square, I997, fig. I) ou de Metal Gear Solid II (Konami, 200I, fig. 2), sont alors inscrites dans une généalogie qui regroupe l'écran

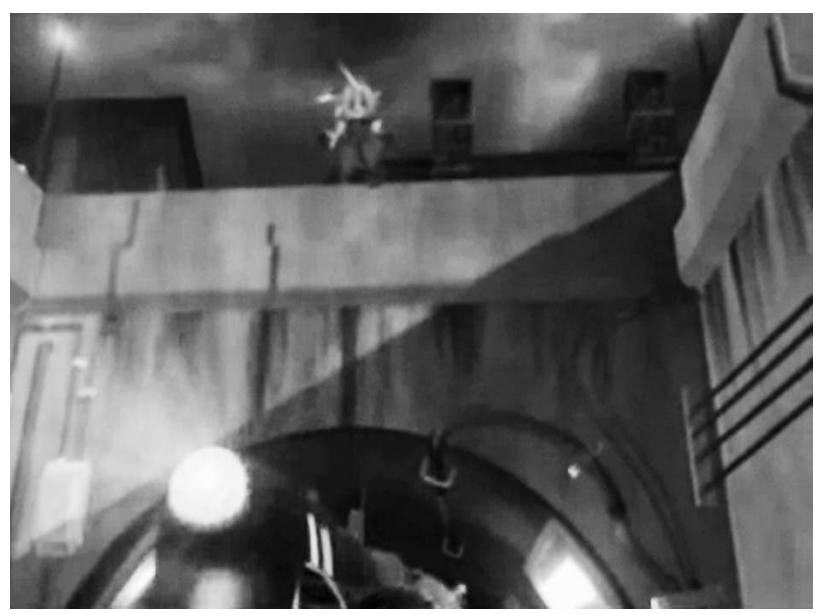

1/ Final Fantasy VII (Square, 1997)
20 James Newman, Videogames, Londres/New York, Routledge, 2004, pp. 72-76. La «cinématique» constitue un exemple de «réponse» informatique particulièrement longue, comparativement à l'animation d'un personnage déclenchée par une action basique comme le saut, le tir ou la course.

21 Rune Klevjer, "In defence of cutscenes », dans Frans Mäyrä (éd.), Computer Games and Digital Cultures Conference Proceedings, Tampere, Tampere University Press, 2002.

22 La recrudescence de travaux portant sur les «cinématiques» durant les années 2000 tient au fait que ces séquences constituaient alors un "sujet de controverse» au sein des communautés de joueurs, mais aussi au sein de la communauté de recherche, dans la mesure où elles cristallisent un ensemble d'enjeux relatifs à la narration vidéoludique et au caractère représentationnel du médium - questions qui figurent parmi les principales lignes de démarcation du champ lors de sa phase initiale de constitution (1997-2006). Au sujet du caractère controversé des cinématiques, voir Simon Egenfeldt-Nielsen, Jonas Heide Smith et Susana Pajares Tosca (éd.), Understanding Video Games: The Essential Introduction, New York, Routledge, 2008, p. 176; James Newman, Videogames, op. cit., p. 93. 


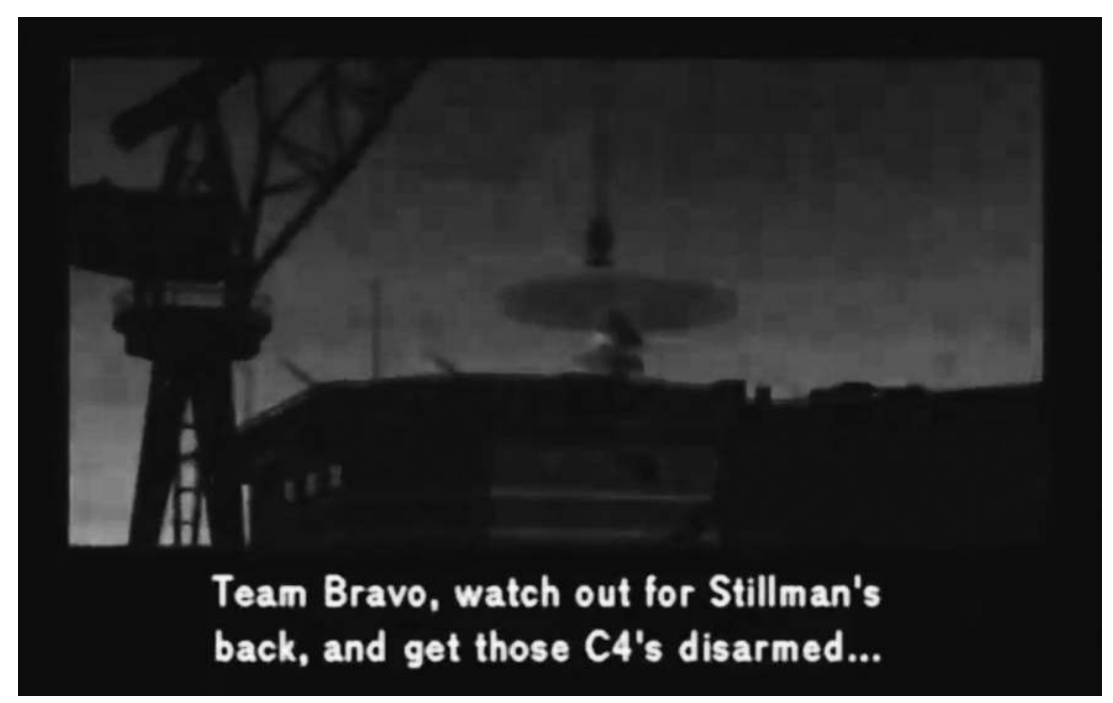

2/ Metal Gear Solid II (Konami, 2001)

d'ouverture de Pac-Man (Namco, I980, fig.3), la séquence introductive de Kung-Fu Master (Irem Corp., I984, fig. 4), ou encore l'écran d'accueil de Balloon Kid (Nintendo, I990, fig. 5).

Pour Newman, Perron et Arsenault, ou encore Blanchet, c'est la facture visuelle des «cinématiques» qui permet de les distinguer des séquences non interactives qui les précèdent historiquement. C’est ainsi par leur emploi «de procédés cinématographiques et télévisuels» que l'auteur Des Pixels à Hollywood peut différencier les cinématiques des séquences non interactives présentes dans les jeux vidéo des années I980. Blanchet propose par ailleurs de qualifier ces séquences de «protocinématiques», tout en précisant que leurs caractéristiques formelles (uniponctualité, immobilité du «cadre», etc.) les rapprochent du cinéma des premiers temps.

Si les approches formelles permettent de mettre à jour les fonctions - aussi bien ludiques que narratives - des séquences non interactives du jeu vidéo, tout en constituant un modèle pertinent pour aborder certaines questions centrales de l'étude du jeu vidéo (à l'instar des processus de rétroaction, ou de la variabilité des postures du joueur face au jeu), elles ont cependant tendance à négliger le caractère proprement textuel 


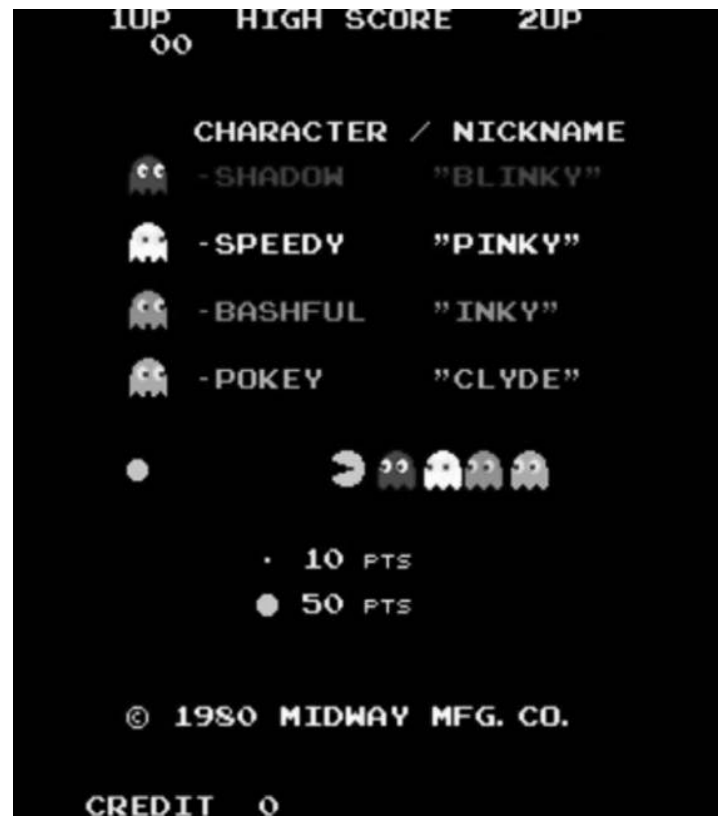

3/ Pac-Man (Namco, 1980)

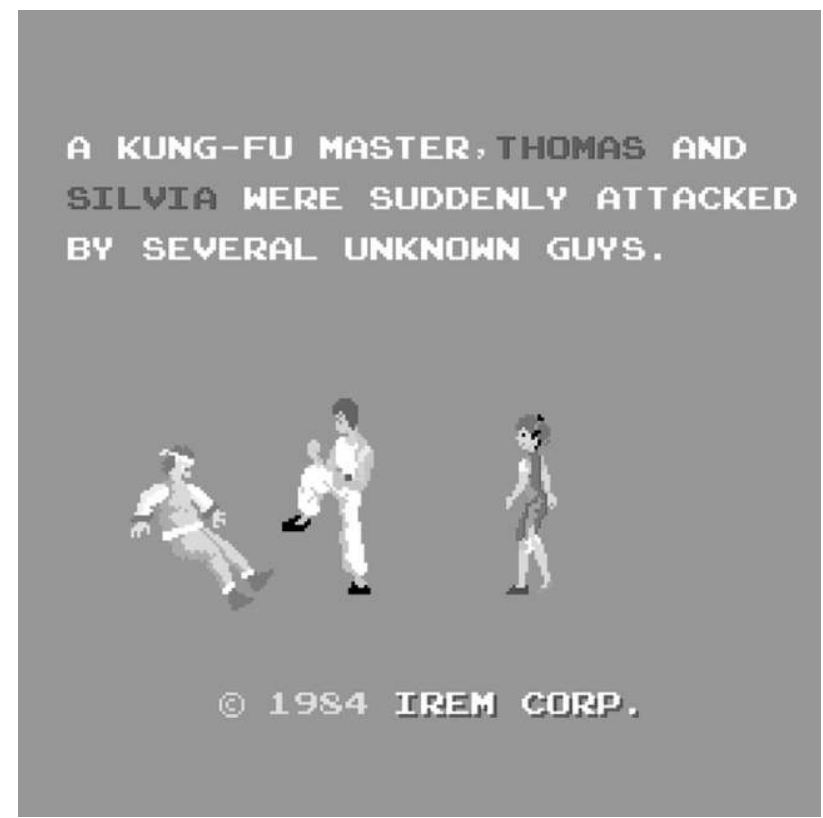

4/ Kung-Fu Master (Irem Corp., 1984)
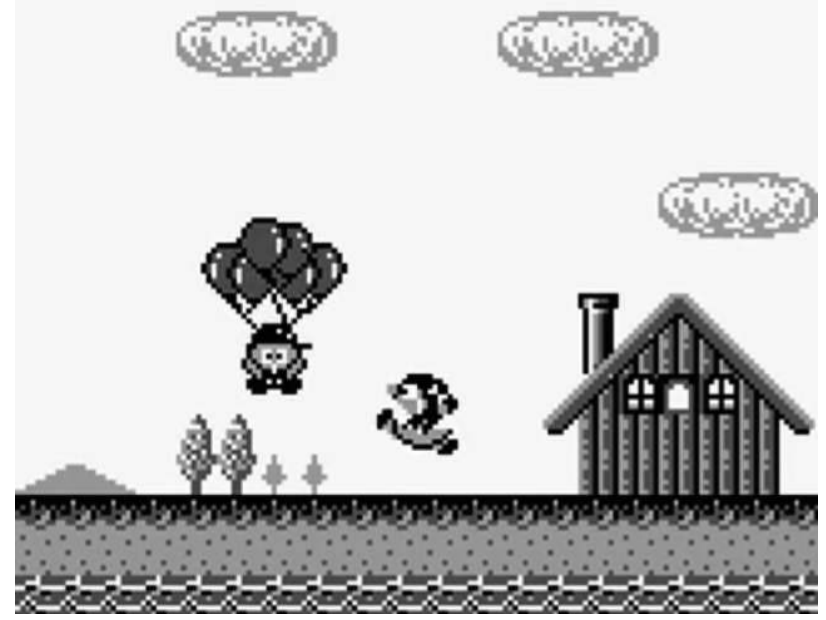

5/ Balloon Kid (Nintendo, 1990) 
23 L'expression, empruntée à la sociolinguistique, est employée dans le domaine du jeu vidéo par Bernard Perron, Hugo Montembeault, Andréane Morin-Simard et Carl Therrien, "The Discourse Community's Cut: Video Games and the Notion of Montage», op. cit., à paraître en $20 \mathrm{I} 8$.

24 François Albera et Maria Tortajada, «Le dispositif n'existe pas!», dans François Albera et Maria Tortajada (éd.), Ciné-dispositifs: spectacles, cinéma, télévision, littérature, Lausanne, L’Age d'Homme, 20II, p. 27.

25 Id., p. 29.

26 Le terme est parfois orthographié «cut-scene». des notions qu'elles engagent. A ce titre, de telles approches ont tendance à concevoir la «cinématique» comme une figure qu'on peut localiser dans les jeux vidéo, avant de la considérer comme un fait de discours, dont l'apparition et la naturalisation se sont jouées dansles textes.

Nous proposons ici de considérer la cinématique - au même titre que l'ensemble des termes employés dans le paratexte vidéoludique pour qualifier les séquences non interactives - comme un fait de discours ayant connu un processus de naturalisation au sein des «communautés discursives ${ }^{23}$ du jeu vidéo. Penser la «cinématique» comme une entité avant tout discursive nous amène alors à une étude des discours de production et de réception, afin de saisir l'évolution des cadres d'intelligibilité qui accompagnent la création, la consommation et la diffusion des jeux vidéo.

Dans ce contexte, nous suspendons les questions d'intermédialité et de remédiation, qui ont été maintes fois traitées par la recherche en game studies, au profit d'une analyse textuelle qui vise à retracer l'évolution de la place du cinéma dans le «cadrage» collectif du jeu vidéo. Cette approche est redevable du cadre méthodologique établi par Maria Tortajada et François Albera autour de la notion de «dispositif» et de son réaménagement dans le contexte de l'histoire du cinéma et de l'audiovision ${ }^{24}$. En effet, nous proposons de considérer la notion de «cinématique» comme une notion clé du lexique vidéoludique. Comme le note les auteurs lausannois, «la notion-type a une histoire, qui est celle de sa 〈fabrication `: il faut donc historiciser et dénaturaliser son emploi» ${ }^{25}$. C'est précisément l'histoire de la «fabrication» de la notion de «cinématique» que nous proposons de retracer à travers une analyse quantitative des discours de réception critique du jeu vidéo du début des années I980 à la fin des années I990. Il s'agira dès lors de saisir l'évolution des termes employés pour qualifier les séquences non interactives du jeu vidéo, mais aussi de repérer les «termes associés » à ces notions clés («cinématique», «cutscene» ${ }^{26}$, «intermission») afin de mieux cerner l'histoire des cadres d’intelligibilité qui accompagnent la diffusion du jeu vidéo.

\section{Méthodologie}

Si l'analyse du discours a longtemps occupé une position marginalisée dans le domaine de l'étude du jeu vidéo, elle connaît un regain d'intérêt considérable depuis quelques années. Ainsi, de nombreux travaux récents ont tiré profit des vastes répertoires en ligne de magazines spé- 
cialisés, de manuels de jeux, ou encore de manuels techniques à l'attention des exploitants de bornes d'arcade, afin d'offrir de nouvelles sources à l'étude du jeu vidéo. Ces projets de recherches dépendent bien souvent des initiatives d'archivage qui sont menées par des communautés de passionnés ayant à cour de créer des bases de données exhaustives de jeux vidéo et de matériel paratextuel.

Un projet mené par le laboratoire d'études vidéoludiques de l'Université de Montréal a ainsi permis de renouveler notre connaissance de l'histoire des genres vidéoludiques en les interrogeant à travers les discours ${ }^{27}$, alors qu'une étude de Graeme Kirkpatrick conduite à partir d'échantillons de la presse spécialisée britannique des années I980 retrace la constitution d'une culture vidéoludique à travers l'émergence d'un lexique partagé témoignant de la consolidation d'une communauté de goûts et de pratiques ${ }^{28}$. Chez Jaakko Suominen, ce sont les logiques générationnelles de nostalgie et de mémoire, au cœur de la culture vidéoludique, qui sont interrogées à travers les discours de presse ${ }^{29}$. Autant de perspectives de recherche qui témoignent de la richesse de l'analyse de discours dans un contexte où la presse spécialisée contribue à forger et à diffuser les notions qui régissent les catégories de perception partagées, en jouant un double rôle «d'arbitre et de médiateur» ${ }^{30}$.

Les travaux de recherche cités supra procèdent majoritairement au dépouillement «à la pièce» des sources sélectionnées, parfois en extrayant des «échantillons» des magazines afin de réduire la quantité d'information à traiter. Les méthodes de reconnaissance optique de caractères (abrégé «OCR» pour «Optical Character Recognition») sont relativement peu employées du fait de la fragilité des résultats obtenus ${ }^{31}$. Effectivement, les maquettes complexes et souvent bariolées des magazines spécialisés tendent à réduire drastiquement l'efficacité des outils d'OCR. Si une partie considérable des ressources disponibles aujourd'hui en ligne ont été passées au crible d'outils d'OCR - c'est le cas de l'impressionnante collection de magazines conservée sur archive.org par l'Internet Archive--, les résultats sont malheureusement inutilisables du fait de leur faible précision.

Dans le cadre de cette recherche, nous avons utilisé un outil récent de reconnaissance de caractères, Vision API ${ }^{32}$ de Google, qui constitue

27 Voir par exemple Carl Therrien, «Inspecting Video Game Historiography
Through Critical Lens: Etymology of the First-Person Shooter Genre», Game
Studies, vol. $15, \mathrm{n}^{\circ}$ 2, décembre 2015. Disponible en ligne: http://gamestudies. org/I502/articles/therrien.

28 Graeme Kirkpatrick, The Formation of Gaming Culture: UK Gaming Magazines, I98I-I995, op. cit., p. 22.

29 Jaakko Suominen, «Return in Play: The Emergence of Retrogaming in Finnish Computer Hobbyist and Game Magazines from the I980s to the 2000s", Kinephanos, Cultural History of Video Games Special Issue, 2015. Disponible en ligne: www.kinephanos.ca/2015/ emergence-of-retrogaming/. Voir également la thèse de Selim Krichane qui propose de cartographier l'émergence de la notion de «caméra» dans le paratexte vidéoludique à partir d'un corpus de magazines spécialisés et de notices d'emploi. Selim Krichane, La Caméra imaginaire. Histoire et théorie des modes de visualisation vidéoludique, thèse de doctorat soutenue à l'Université de Lausanne en mai 2017.

30 Graeme Kirkpatrick, The Formation of Gaming Culture, op.cit., p. 53.

31 Carl Therrien, «Inspecting Video Game Historiography Through Critical Lens: Etymology of the First-Person Shooter Genre», op. cit., sans pagination.

32 Cette interface de programmation pour la reconnaissance d'images, qui appartient à la suite d'outils en ligne «Google Cloud», a été lancée en 20I7. La plateforme «Google Cloud» permet depuis 2oIr d'exécuter à distance, sur les serveurs de Google, diverses applications proposées par cette entreprise, allant du stockage de données à la traduction de textes. Pour plus d'informations, voir le site de Vision API (https://cloud. google.com/vision/) et le site de «Google Cloud»(https://cloud.google.com/). 
33 Antonio Casilli, «Digital Labor: travail, technologies et conflictualités. Qu'est-ce que le digital labor?», Editions de l'INA, pp. I0-42, 2015. Disponible en ligne: https://halshs.archives-ouvertes. fr/halshs-ori457ir.

34 Principalement les langages Bash et Python. Les scripts que nous avons rédigés et exécutés sont inspirés des exemples publiés à l'adresse https:// googlecloudplatform.github.io/googlecloud-python/latest/vision/index.html. une alternative aux logiciels employés traditionnellement (principalement Adobe Acrobat Pro et ABBYY FineReader). Vision API a l'avantage de bénéficier des corpus colossaux amassés au fil du temps par Google afin d'«entraîner», suivant le terme en vigueur, ses algorithmes de reconnaissance d'image. En amont de la tâche même de lecture des caractères, l'application opère une série de transformations permettant ensuite de repérer avec une plus grande efficacité les zones de textes et de les décrypter. Après cette extraction des chaînes de caractères, le logiciel utilise des fonctionnalités qui lui permettent d'extrapoler le choix de termes à partir de la distance d'édition entre un terme lu de manière «brut» et les mots du dictionnaire associé à la langue détectée, tout en prenant en compte la probabilité de co-occurrences des mots en question. L'application fournit également une mesure de précision des résultats, utile notamment lorsque la reconnaissance débouche sur un terme absent du lexique correspondant à la langue identifiée - c'est par exemple souvent le cas des noms propres et des termes techniques.

Ce logiciel bénéficie en outre de l'expertise de l'entreprise américaine en termes de détection et d'annotation d'images, ce qui s'avère efficace pour isoler les éléments de textes dans un corpus qui juxtapose (ou, dans certains cas, superpose) images et textes. Sur la base de quelques cas limites que nous avons soumis à plusieurs logiciels, nous avons observé que les variations chromatiques des fonds, la superposition de motifs et de textes, ou encore l'inclinaison variable des paragraphes (fig. 6) étaient mieux traitées par ce logiciel.

Du côté des désavantages, outre le fait de travailler avec un logiciel propriétaire - une «boîte noire» - dont la précision est en partie le résultat de l'exploitation du digital labor ${ }^{33}$ de millions d'usagers d'internet, on peut retenir sa complexité qui nécessite de la part de l'utilisateur une certaine maîtrise de la programmation informatique ${ }^{34}$. Effectivement, Vision API ne dispose pas d'une interface graphique. Etant donné que notre corpus comporte plusieurs dizaines de milliers de pages de magazines et que l'algorithme traite chaque page comme un «document» autonome, nous avons dû mettre en place une chaîne d’opérations pour télécharger les magazines numérisés, les envoyer aux serveurs «Google Cloud», récupérer les données produites, reconstruire les textes, et finalement construire une interface pour interroger la base de données ainsi obtenue. 


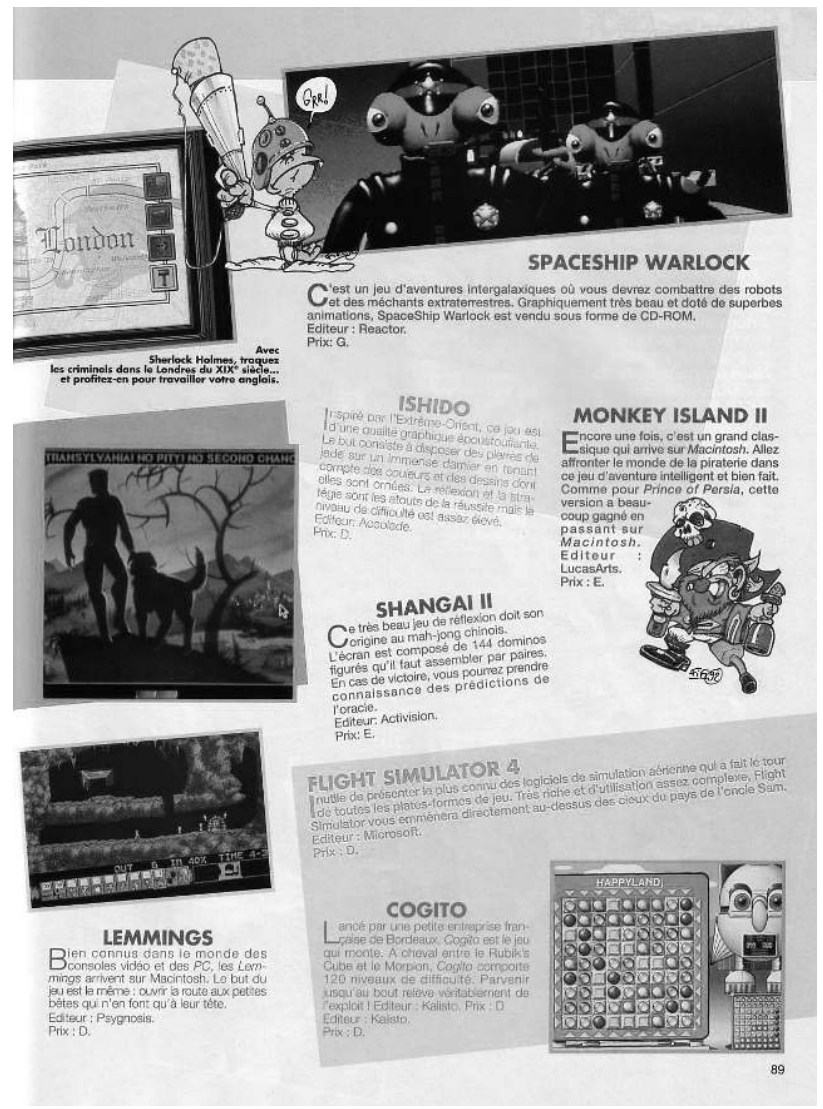

6/ La ligne graphique bariolée et les textes inclinés de nombreux magazines de jeux vidéo compliquent la tâche des logiciels d'OCR (Tilt, n¹10, janvier 1993, p. 89)

Dans le cadre de la présente recherche, nous avons construit notre corpus à partir de quatre magazines «de référence»: deux magazines français, Tilt ${ }^{35}$ et Joystick ${ }^{36}$, un magazine américain, Computer Gaming World (désormais CGW) ${ }^{37}$, et une revue anglaise, Computer and Video Games $(C V G)^{38}$. Ces quatre publications à parution mensuelle nous offrent un large échantillon des pratiques en vigueur au sein des «communautés discursives» du jeu vidéo dans trois zones géographiques distinctes.
35 Tilt était le premier magazine consacré au jeu vidéo en France. Edité par Editions Mondiales S.A., on dénombre I22 numéros parus entre septembre 1982 et janvier 1994, d'abord à un rythme bimestriel, puis mensuel. Le magazine traitait des jeux vidéo disponibles pour les micro-ordinateurs. Tous les numéros de Tilt sont inclus dans notre corpus.

36 Joystick est un magazine mensuel lancé en janvier I99o qui couvrait les jeux vidéo sortis sur toutes les plateformes du marché (micro-ordinateurs et consoles) avant de se consacrer uniquement aux jeux vidéo disponibles sur des micro-ordinateurs dès mars 1993 $\left(n^{\circ} 36\right)$. Le magazine a d'abord appartenu au Groupe Sipress, puis à Hachette Presse dès juin $1993\left(n^{\circ} 39\right)$ et jusqu'en 2003 (le magazine a cessé de paraître en 20I2). Les numéros de janvier 1990 ( $\left.{ }^{\circ} \mathrm{I}\right)$ à décembre $1998\left(n^{\circ} 99\right)$ sont inclus dans notre corpus.

37 Computer Gaming World commence à paraître en novembre I98I de manière bimestrielle jusqu'en I985, puis mensuelle dès i988. Le magazine couvre les jeux vidéo sortis sur des micro-ordinateurs. Il appartient tout d'abord à Golden Empire Publications Inc., puis dès I993 à la compagnie Ziff Davis. Les numéros allant de novembre I98I $\left(\mathrm{n}^{\circ} \mathrm{I.I}\right)$ à décembre 1998 ( $n^{\circ}$ I73) sont inclus dans notre corpus.

38 Le magazine anglo-saxon Computer and Video Games paraît à un rythme mensuel dès novembre I98I. Ses articles de réception critique sont principalement consacrés aux sorties de jeux vidéo sur micro-ordinateurs. Nous avons inclus les numéros allant de novembre i98I $\left(n^{\circ} \mathrm{I}\right)$ à juillet $\mathrm{I} 998\left(\mathrm{n}^{\circ} 200\right)$ dans notre corpus. Le $n^{\circ}$ I62 est manquant. 
40 www.cgwmuseum.org/.

41 https://archive.org/.

42 https://retrocdn.net/.

43 Un comparatif entre les suites d'utilitaires «ImageMagick», "Ghostscript» et «Poppler» a montré que l'utilitaire pdfimages de la suite «Poppler» est significativement plus rapide pour cette tâche et propose les résultats les plus appropriés tout en laissant les images intactes.

44 Les tarifs détaillés sont disponibles à l'adresse suivante: https://cloud. google.com/vision/pricing.

45 Nous avons «consommé» 68437 unités (avec quelques ratages) pour un total de ioo,67 CHF.

46 L'optimisation de la préparation et de l'envoi des données est également à prendre en compte: l'API se base effectivement sur le poids des images pour fixer une limite aux requêtes (ı Mo). Il est donc possible de réduire les coûts d'un facteur io en envoyant les images de manière groupée, mais ceci complique fortement la préparation du script utilisé lors de la transmission des données à «Google Cloud». Nous n'avons pas été en mesure de tester cette solution dans le cadre de la présente recherche.

47 Ces informations peuvent être ensuite superposées au fichier «PDF» d'origine, permettant par exemple de sélectionner du texte et de le copier.
Il existe plusieurs initiatives collaboratives privées visant à préserver les publications anciennes liées au jeu vidéo et à l'informatique. Les numéros de Tilt et Joystick ont pu être récupérés grâce au travail de la communauté réunie autour du site web "Abandonware Magazines » ${ }^{39}$, les numéros de Computer Gaming World grâce au «Computer Gaming World Museum ${ }^{40}$, et les numéros de Computer and Video Games grâce à l'association à but non lucratif «Internet Archive» ${ }^{41}$ et à la communauté qui alimente la base de données accessible en ligne «Retro CDN» ${ }^{42}$. La fonctionnalité permettant d'envoyer des fichiers au format «PDF» à Vision API était encore en phase de développement au moment de cette étude. Lorsque les sources étaient disponibles en «PDF», nous avons converti les documents au format «JPEG» grâce à l'utilitaire pdfimages ${ }^{43}$.

L'envoi des fichiers images aux serveurs «Google Cloud» et leur traitement est l'étape la plus coûteuse - en temps comme en argent - de ce protocole d'extraction et d'analyse de données textuelles. Nous avons observé que Vision API était capable de traiter en moyenne une image (c'est-à-dire une page de magazine) par seconde. Cependant, son modèle économique dépend du nombre de requêtes nécessaires: extraire le texte d'une image équivaut à une unité, mille unités coûtent I,50 \$ (soit I,50 CHF), et les Iooo premières unités du mois ne sont pas décomptées ${ }^{44}$. Avec un capital de 300 \$ (virtuels) «offerts» une seule fois, au moment de l'inscription, notre utilisation des services «Google Cloud» n'a pas induit de coûts ${ }^{45}$. Cela dit, le traitement d'un corpus plus grand (composé, par exemple, d'une dizaine de magazines) nécessitera un budget conséquent - paramètre à prendre en compte par celles et ceux qui souhaitent effectuer une recherche similaire ${ }^{46}$.

Pour chaque page envoyée, l'application produit un fichier contenant des données au format «JSON», un format de structuration de données communément utilisé aujourd’hui. Ce fichier est composé du texte entier reconstitué, mais aussi des coordonnées dans l'image des mots détectés ainsi que des paragraphes ${ }^{47}$. Chaque élément de texte est accompagné d'un score de confiance calculé par le logiciel en fonction des conditions techniques du repérage (contraste, netteté, alignement du texte, etc.), de la plausibilité de son existence, et de sa probabilité d'apparition à proximité des autres termes détectés.

Les résultats obtenus ont démontré l'efficacité de cet outil en comparaison des solutions habituellement utilisées dans le domaine de la recon- 
naissance de caractères destinées à la presse populaire. En effet, la comparaison de plusieurs échantillons atteste la qualité des résultats pour les magazines Tilt, Joystick ${ }^{48}$ et Computer Gaming World ${ }^{49}$. Le principal problème rencontré par Vision API avec les textes soumis est la reconnaissance de la mise en pages. En effet, un texte sur deux colonnes est fréquemment lu en passant d'une colonne à l'autre de manière continue. En l'occurrence, il ne s'agit pas de reconstruire exactement le texte: l'ordre exact des mots n’importe pas pour évaluer la fréquence d'usage et la répartition des occurrences d'un mot dans le temps (l'analyse détaillée des usages étant ensuite conduite à partir d'une lecture rapprochée). Cela dit, lorsque les colonnes de texte ne sont pas identifiées, les césures ne sont pas corrigées. Par conséquent, certains termes échappent à la détection automatique.

Le traitement des textes et le calcul des distributions d'occurrences de mots se sont faits avec le langage de programmation $R^{50}$ et le logiciel Rstudio ${ }^{51}$, des outils libres et répandus pour l'analyse de données. Nous avons isolé tous les mots présents dans les magazines (y compris ceux comportant des erreurs de reconnaissance de caractères), puis nous les avons comptés et répartis dans le temps en fonction des dates de sortie des magazines. Il est ensuite possible en entrant un mot sous la forme d'une expression régulière ${ }^{52}$, d'afficher la répartition de ses apparitions dans le temps. Les scripts développés pour ces tâches ainsi que les

48 Le site Abandonware Magazines propose quelques articles numérisés et corrigés à la main. Une comparaison sur la base des tests de Croisière pour un cadavre (Delphine Software, I99I, disponible dans Tilt, $\mathrm{n}^{\circ}$ 93) et d'Alone in the Dark 2 (Infogrames, I994, disponible dans Joystick, $\mathrm{n}^{\circ} 44$ ), montrent que les textes que nous avons obtenus avec $\mathrm{Vi}$ sion API et les textes vérifiés sont quasiment identiques. Nous nous référons aux valeurs mesurées avec la similarité cosinus, une mesure de distance entre textes variant entre o (lorsqu'ils sont identiques) et I (lorsqu'ils n'ont aucun terme en commun). Pour le texte de Croisière pour un cadavre - composé de 556 mots -, elle est égale à 0,003 , tandis que pour le texte de Alone in the Dark 2 - composé de II43 mots - elle est égale à o,0o8ı. Ces textes sont consultables en ligne sur www.abandonware-magazines.org/. Notons que les logiciels d'OCR se révèlent parfois zélés et extraient fréquemment le texte contenu dans les illustrations. Nous avons retiré ces éléments pour réaliser cette comparaison.

49 Dans le cas de CGW, un expert a corrigé les textes des Ioo premiers numéros de ce magazine à la ligne graphique plus proche de celles de Tilt et de Joystick que de CVG. Les textes corrigés «à la main» sont disponibles sur le site du «Computer Gaming World Museum». Une com- paraison entre ces textes corrigés et les textes que nous avons obtenus après $\mathrm{OCR}$ (pour les roo premiers numéros du magazine) donne d'excellents résultats, avec des valeurs oscillant entre o,0016 et o,02 pour des valeurs médiane et moyenne égales à o,oo9. Les différences entre les textes s'expliquent en partie par les erreurs d'OCR, mais aussi par l'intégration des textes des publicités et des captures d'écran dans les versions transcrites automatiquement (alors que ces éléments textuels ont été retirés manuellement de la version transcrite par l'expert). $\mathrm{Ne}$ disposant pas de transcriptions réalisées à la main pour CVG, nous n'avons pas été en mesure d'évaluer la performance de l'OCR pour ce magazine.

50 Pour plus d'informations sur ce langage de programmation, voir le site de la « R Foundation for Statistical Computing»: www.r-project.org/.

51 Le logiciel est disponible en ligne à l'adresse suivante: www.rstudio.com/.

52 Les expressions régulières permettent de soumettre à l'algorithme un mot ainsi que ses variantes orthographiques. Par exemple, pour chercher l'expression «séquence cinématique», on entrera «s[eé] quence $[\mathrm{s}]^{*} \operatorname{cin}[$ eé $]$ matique $[\mathrm{s}]^{*}$ ». Les crochets signifient que l'algorithme ne doit choisir qu'un seul caractère parmi ceux qu'ils contiennent (dans ce cas, soit le «e», soit le «é»). L'étoile signifie que le (ou les) caractère(s) à l'intérieur des crochets sont facultatifs. Dans cet exemple, la requête englobe les occurrences de l'expression «séquence cinématique» au singulier comme au pluriel ainsi que les termes où les signes diacritiques n'ont pas été détectés par le logiciel de reconnaissance optique de caractères - une situation fréquente en pratique. 
53 L’ensemble des données récoltées dans le cadre de ce projet sont disponibles à l'adresse suivante: https:/github.com/ yrochat/Decadrages.

54 Dominic Arsenault et Vincent Mauger, «Au-delà de «l'envie cinématographique >: le complexe transmédiatique d'Assassin's Creed», Nouvelles vues, revue sur les pratiques et les théories du cinéma au Québec, $\mathrm{n}^{\circ} \mathrm{I}$, hiver-printemps 2012, pp. 3-4 [version en ligne]. Disponible en ligne: www.nouvellesvues.ulaval.ca/ index.php?id=2432.

55 «This scene in particular accomplishes a great deal in suggesting the cinematic flavor that the designers intended and is great fun to watch.» (CGW, $\mathrm{n}^{\circ} 34$, janvier I987, p. 69).

$56 \mathrm{CGW}, \mathrm{n}^{\circ} 38$, juin I987, p. 6.

57 Id., p. 48.

$58 \mathrm{CGW}, \mathrm{n}^{\circ} 45$, mars i988, p. i6. Il semblerait que le jeu traité dans cet article prévisionnel ne soit jamais sorti. Il est également possible qu'il soit sorti sous un autre nom. résultats de la reconnaissance optique de caractères - sur les 605 magazines de notre corpus - sont disponibles en ligne ${ }^{53}$. A partir de ces outils et de la méthode d'extraction de données textuelles présentée supra, nous avons pu rechercher les termes utilisés pour désigner les séquences non interactives des jeux vidéo dans l'ensemble de notre corpus afin de retracer l'histoire de la notion de «cinématique».

\section{Cinématique/Cinematic: histoire d'une notion}

Si le terme de «cinématique» est parfois perçu comme une exception française, son histoire est très directement liée à l'anglais, où le terme «cinematic» - qui signifie «cinématographique»- a été très régulièrement convoqué par la presse spécialisée dès la seconde moitié des années I980.

Une des premières occurrences du terme apparaît dans le numéro de janvier 1987 du magazine CGW au sujet du jeu Defender of the Crown (1986) de la compagnie Cinemaware, qui, comme son nom l'indique, témoigne d'une «envie cinématographique ${ }^{54}$ considérable visant à créer des jeux vidéo qui puisent dans les ressources formelles du cinéma, faisant du studio un des représentants emblématiques du genre des «films interactifs» qu'il contribue à instituer. Lorsque le journaliste commente la séquence de rencontre amoureuse qui succède à une séquence de duel à l'épée - à la condition que le joueur soit victorieux -, il ajoute: «Cette scène joue un rôle important dans l'établissement de la 〈touche cinématographique〉 que les développeurs souhaitaient établir et est particulièrement sympathique à regarder ${ }^{55}$. Le journaliste parlera ensuite à deux reprises de l'«écrin cinématographique» du jeu [«cinematic vehicle»], reconduisant ainsi le discours promotionnel des jeux Cinemaware qui sont construits et promus comme des «films interactifs » dès la sortie de Kings of Chicago en I986.

Dans CGW, l'utilisation de l'adjectif «cinematic» se généralise durant la seconde moitié des années i980 (fig.7). Les jeux d'aventure, les jeux de rôle et les simulations de vol suscitent ainsi régulièrement l'emploi de l'adjectif «cinématographique». En I987, par exemple, un journaliste évoque l'«introduction cinématographique» ${ }^{56}$ [ «cinematic introduction»] du jeu SDI (Sega, 1987), alors que Borrowed Time (Interplay, 1985) est qualifié d'«aventure graphique magnifiquement cinématographique » ${ }^{57}$ [«superbly cinematic graphic adventure»]. En I988, un critique considère que les graphismes du jeu de rôle Citadel (Mindscape) témoignent d'une «influence cinématographique ${ }^{58}$ [ “inematic influences $)$, alors que l'un 


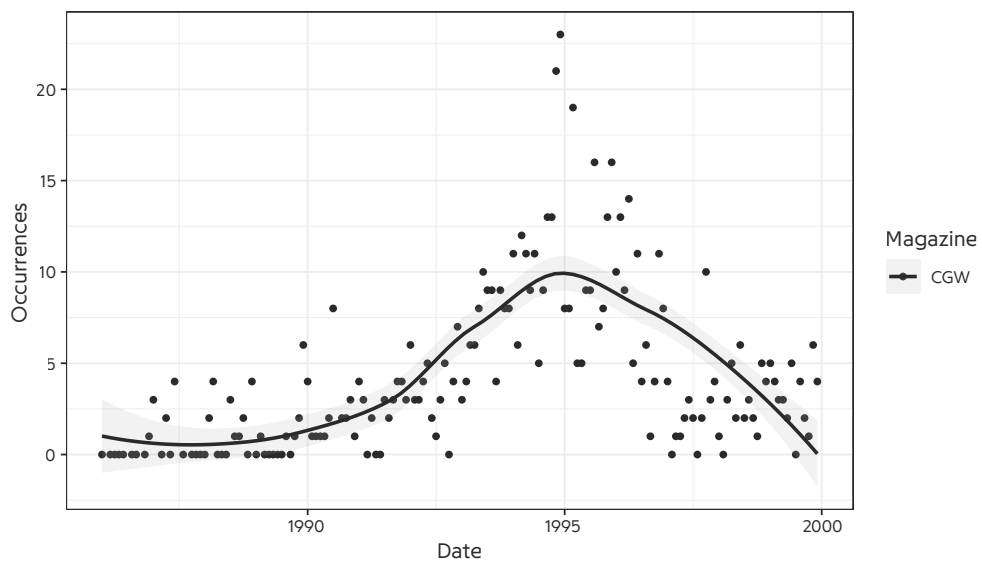

de ses collègues, de retour du «Winter Consumer Electronics Show», indique qu'une tendance majeure de l'industrie consiste à offrir des «angles de vue cinématographiques» [«cinematic perspectives»] ${ }^{59}$.

Dans le numéro de décembre 1989 du magazine américain débute une nouvelle série d'articles rédigés par Charles Ardai ${ }^{60}$ concernant le genre des «films interactifs», alors en vogue au sein de l'industrie dominante. Dans une visée téléologique - et relativement péremptoire -, Ardai indique en ouverture de son premier article (dédié à David Wolf: Secret Agent, Dynamix, 1989), que «dès le départ, les jeux d'ordinateur ont cherché à atteindre un style et un degré de raffinement qu'on peut

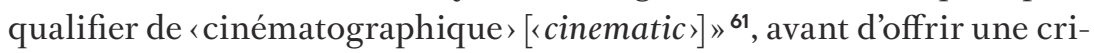
tique très acerbe de ce «film interactif» quant à son manque d'interactivité et la faiblesse de son scénario ${ }^{62}$. Sur les i28 occurrences du terme

59 CGW, no 44 , février 1988 , p. 36. Voir également l'article dédié à la sortie de King's Quest IV (Sierra On-Line, I988) dans le numéro de CGW de décembre I988, dans lequel on trouve les expressions de «touches cinématographiques», «effets cinématographiques», «approches cinématographiques», «dialogues cinématographiques» (CGW, n ${ }^{\circ}$ 54, décembre I988, pp. I9-2I). L’article est accompagné d'une interview de Roberta Williams, développeuse du jeu en question. En anglais, les expressions employées sont «cinematic touches», «cinematic effects», «cinematic approaches» (Roberta Williams), «cinematic dialogues».
7/ Les occurrences du terme "cinematic » dans Computer Gaming World (de janvier 1986 à décembre 1999, nos 25-185)

60 Ecrivain de science-fiction qui contribue régulièrement à la revue d'Isaac Asimov, Science Fiction Magazine, ainsi qu'au Mystery Magazine d'Ellery Queen.

61 David Ardai, rubrique «Popcorn not included» [référence au slogan des films interactifs de Cinemaware dès I986], CGW, n 66, décembre i989, p.36: «From the start, computer games have aspired to a style and a degree of polish best described as <cinematic $>$.»

62 Ardai inscrit, à l'image des chercheurs que nous avons cités plus haut, les «films interactifs» de la fin des années i980 dans la lignée de jeux antérieurs, comme Ms.Pac-Man, qui utilisaient également des «〈interludes » animées» en guise de ponctuation des niveaux de jeu. Ardai critique la tendance qui voit ces «séquences cinématiques» [«cinematic-sequence»] devenir le clou des jeux en question, au détriment de la jouabilité. Id., p. 36. 
8/ Les occurrences du terme "cinematic sequence » dans Computer Gaming World (de janvier 1989 à décembre 1999, nos 55-185). La substantivation du terme "cinematic » conduit à la disparition du syntagme «cinematic sequence » en 1998
64 «British publisher Palace Software places digitized film clips of various cinematic sequences in order to advance the plot of their multi-player adventure game» (CGW, nº 92, mars i992, p. 26).

65 Id., p. 28. Au sujet de la société Paragon: "Their new fantasy game, Spellbound, features plenty of cinematic sequences with complete digitized voicetrack».

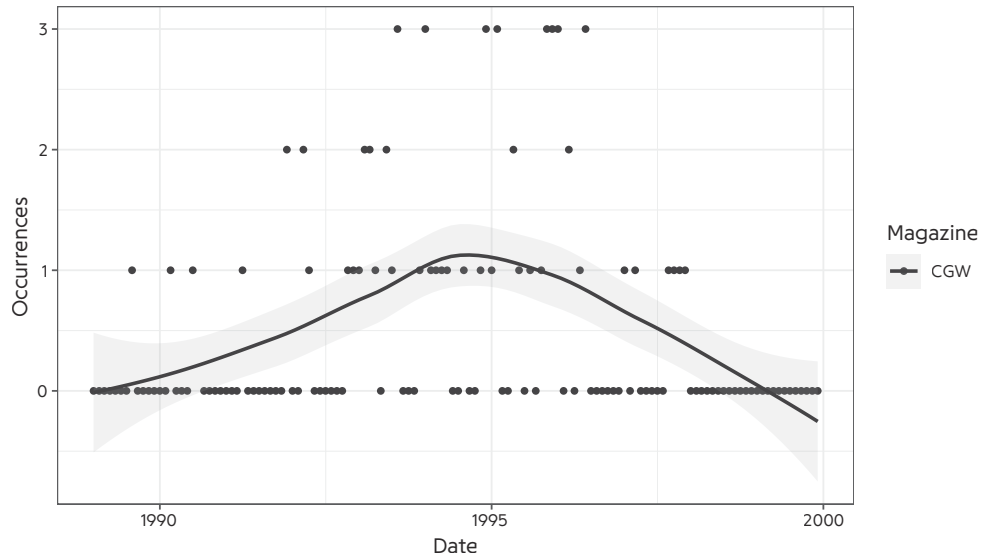

de «cinematic» recensées dans le magazine CGW de I982 à I992, on compte uniquement des emplois de la forme adjectivale du terme et on repère par ailleurs l'apparition progressive de l'expression «séquence cinématique» [«cinematic sequence»] pour qualifier les segments non interactifs des jeux traités, à partir de 1990 ( fig. 8).

En mars 1990, un critique commente par exemple la séquence d'introduction du jeu de rôle Ultima IV: The Quest for The Avatar (Origin Systems, I985) en la qualifiant de «séquence cinématique» ${ }^{63}$. En mars i992, CGW offre une liste de jeux qui utilisent des «images de films numérisées» [digitized film footage] en guise de matériau vidéoludique. Le journaliste indique alors que l'éditeur britannique Palace Software utilise dans son dernier jeu des extraits de films numérisés issus de "différentes séquences cinématographiques [cinematique sequences]» ${ }^{64}$ à des fins narratives. Plus loin, au sujet du dernier jeu du studio américain Paragon, un critique indique que Spellbound (qui sortira sous le nom de Challenge of the Five Realms, MicroProse, I992) contient «de nombreuses séquences cinématographiques [cinematic sequences] avec une piste-voix complètement numérisée» ${ }^{65}$. La présence croissante d'images en prise de vues réelle intégrées dans des jeux vidéo au début des années 1990 explique l'usage fréquent de l'expression «cinematic sequence», pouvant dès lors qualifier des segments non interactifs de divers jeux. 
Dans le magazine britannique CVG, l'expression «cinematic sequence» apparaît dans le courant de l'année i992. Dans le numéro de décembre 1992 , un critique évoque alors les «séquences cinématographiques d'action» ${ }^{66}$ qui ponctuent le simulateur de combat spatial de LucasArts, $X$-Wing (1993), adaptation vidéoludique issue de la franchise Star Wars. Une deuxième occurrence apparaît dans la critique du portage de Another World (Delphine Software, I99I) pour la Super Nintendo, alors que le critique évoque avec enthousiasme la «séquence cinématographique» [«inematic sequence»] ${ }^{67}$, qui fait office d'introduction au jeu. Les deux jeux en question s'inscrivent par ailleurs dans la catégorie des productions pour lesquelles le cinéma joue un rôle de modèle décisif et guide avec force leur conception ainsi que leur caractérisation auprès de la presse et du public.

\section{Traduction fautive : « séquence cinématique »}

La traduction française de l'expression «cinematic sequence» constitue une des voies d'entrée de la notion de «cinématique» dans le paratexte francophone du jeu vidéo. Effectivement, l'adjectif «cinematic» sera allègrement transposé en français sous la forme adjectivale «cinématique».

Suivant les résultats que nous avons obtenus, il semblerait que ce choix de traduction provienne initialement de la sphère de production

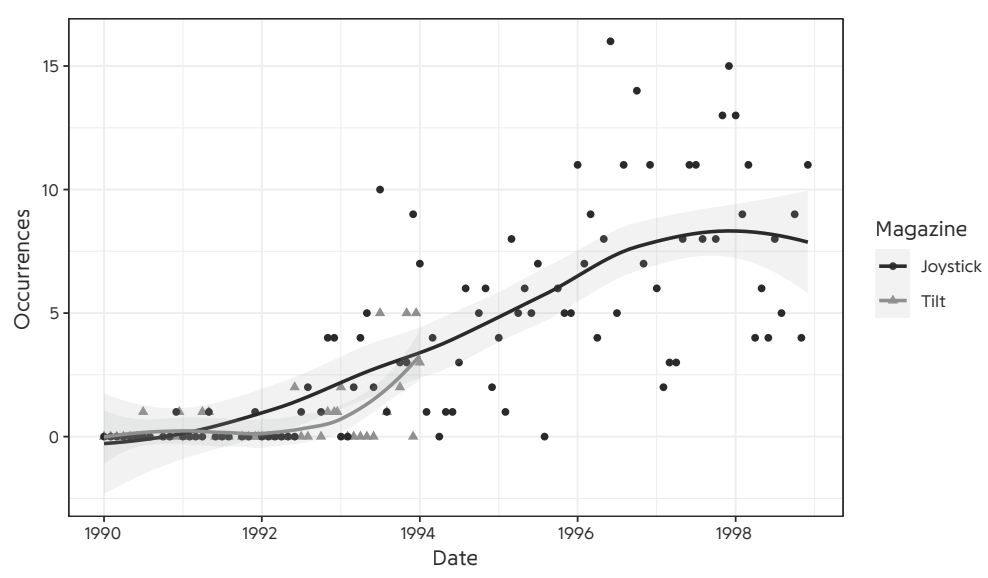

66 CVG, $\mathrm{n}^{\circ}$ 133, décembre 1992, p. 22. Le journaliste évoque les «full screen cinematic action sequences» incluses dans le jeu $X$-Wing.

67 Id., p. 27.
9/ Les occurrences du terme "cinématique » dans Tilt (de janvier 1990 à janvier 1994, $n^{\circ}$ 74-122) et Joystick (de janvier 1990 à décembre 1998, nos 1-99) 
68

Joystick, n² 22, décembre I99I, p.3.

69 Joystick, n 29, juillet I992, p. 25.

70 Joystick, nº 30, août I992, p. I8.

71 Id., pp. 30-3I.

72 Tilt, $\mathrm{n}^{\circ}$ I03, juin I992, p. го.

73 Id., p. 22. et du discours publicitaire, pour ensuite être adopté par les critiques et les joueurs. En décembre i99ı, une publicité de l'éditeur Accolade dans Joystick évoque, par exemple, l'«incroyable effet cinématique ${ }^{68}$ provoqué par l'utilisation d'images numérisées intégrées aux graphismes du jeu d'aventure Les Manley in: Lost in L.A. (Perdu dans L.A., Accolade, I99I), par ailleurs qualifié de «film interactif». On repère en outre un emploi régulier de cet adjectif au sein de la presse spécialisée francophone à partir de la fin de l'année 1992 (fig. 9). Une publicité de l'éditeur Electronic Arts parle alors de «briefings cinématiques» ${ }^{69}$ au sujet du simulateur de vol Chuck Yeager's Air Combat (Electronic Arts, I99I), alors que, dans le numéro suivant de Joystick, un journaliste vante les «écrans cinématiques ${ }^{70}$ du jeu Challenge of the Five Realms. Le premier article de Joystick dédié au jeu Mantis (Paragon Software, 1992) - clone de Wing Commander (Origin Systems, 1990) - évoque quant à lui les «séquences cinématiques» qui ponctuent le jeu, également qualifiées de «séquences intermédiaires ${ }^{71}$. En juin 1992 , un journaliste du magazine français Tilt parle d'«animations spectaculaires de type 〈cinématique»» ${ }^{72}$ au sujet du jeu d'aventure Johnny Crash, alors que dans le même numéro, la démo de Alone in the Dark (Infogrames, 1992) présentée en avant-première au «European Computer Trade Show» (ECTS) à Londres amène un journaliste à qualifier les «effets visuels» du jeu (produit par son «système de caméra») de «très cinématiques» ${ }^{73}$.

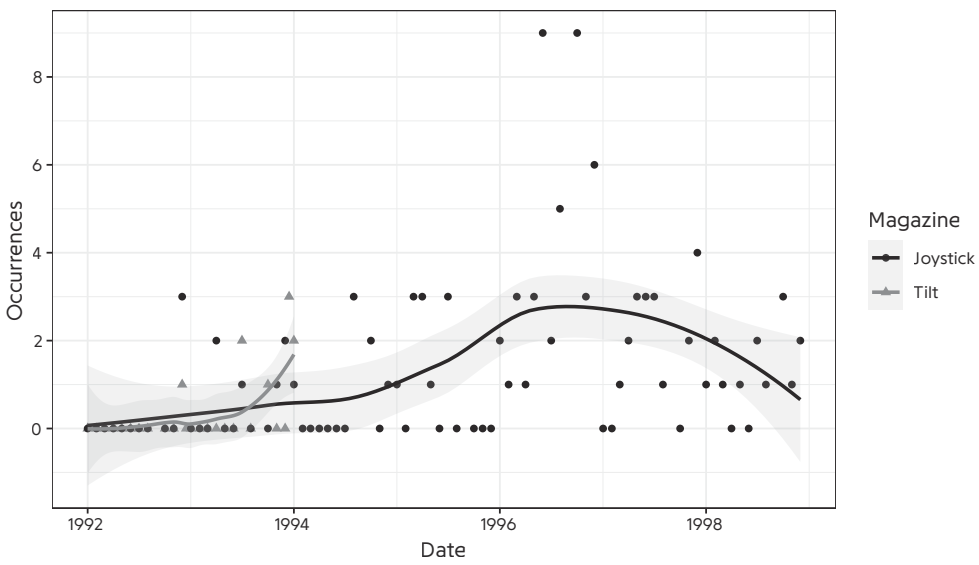

10/ Les occurrences du terme "séquence cinématique» dans Tilt (de janvier 1992 à janvier 1994, nos 98-122) et Joystick (de janvier 1992 à décembre $1998, n^{\circ 5} 23-99$ ) 


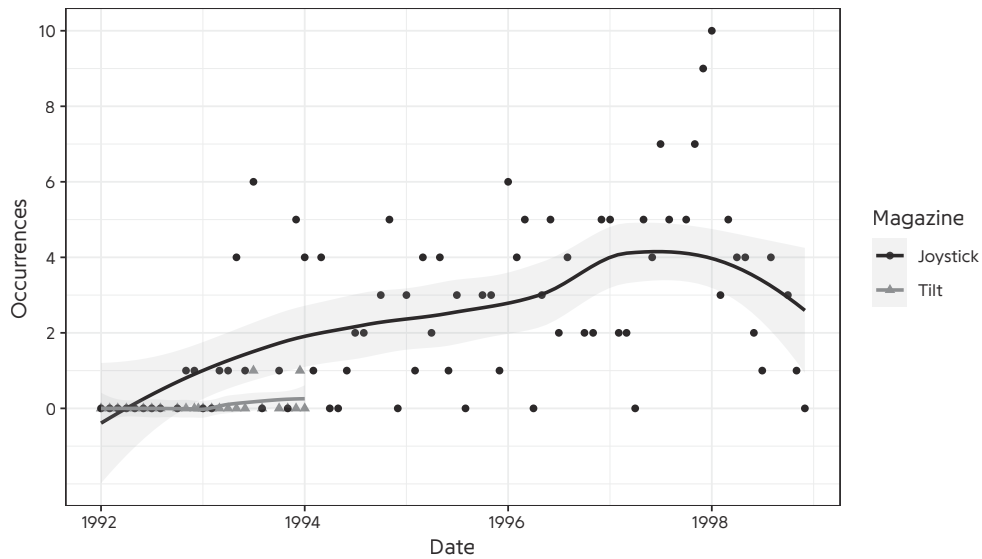

Dans la presse spécialisée francophone, l’adjectif «cinématique» apparaît alors majoritairement, à partir de la fin de l'année i992, au sein du syntagme figé «séquence cinématique» (ou, alternativement, «scène cinématique», fig. IO-II). Le terme est donc employé dès le début des années I990 afin de qualifier les séquences non interactives des jeux vidéo. Cela dit, durant cette période, la notion est uniquement utilisée dans sa forme adjectivale, et qualifie majoritairement les termes de «scène» ou «séquence». Cet usage est d'ailleurs facilité par le discours de production. D’une part, le terme «cinematic» est régulièrement employé par les éditeurs et les studios qui s'inscrivent dans cette tendance à la «cinématographicité» visible dans les jeux d'aventure (Sierra On-Line, LucasArts, Delphine), les jeux de rôle (SSI) ou encore les simulations de vol dès la seconde moitié des années i980. D’autre part, le discours publicitaire, présent dans ces magazines, favorise, comme nous l'avons vu, l'emploi du terme «cinématique». Ainsi, une publicité pour le jeu de pilotage Comanche: Maximum Overkill (NovaLogic, I992) - qui vante par ailleurs les mérites des "animations cinématiques » ${ }^{74} \mathrm{du}$ jeu - parue dans le magazine Tilt (décembre I992) mobilise parmi ses citations de presse l'extrait d'un article d'un numéro antérieur du même magazine, illustrant de ce fait la circularité des discours au sein du paratexte vidéoludique ainsi que la récupération du discours de «glorification technoindustrielle ${ }^{75}$ de l'industrie par la presse.
11/ Les occurrences du terme «scène cinématique» dans Tilt (de janvier 1992 à janvier 1994, nºs 98-122) et Joystick (de janvier 1992 à décembre 1998, nos 23-99)

74 Tilt, $\mathrm{n}^{\circ}$ Io9, décembre I992, p. 85.

75 Carl Therrien et Martin Picard, «Techno-industrial celebration, misinformation echo chambers, and the distortion cycle. An Introduction to the History of Games International Conference proceedings», Kinephanos. Revue d'études des médias et de culture populaire, numéro spécial, janvier 20I4. Disponible en ligne sur: www.kinephanos.ca. 
12/ Les occurrences du terme "cutscene» dans Computer Gaming World (de janvier 1987 à décembre 1999, $\mathrm{n}^{\circ \mathrm{s}}$ 34-185) et Computer and Video Games (de janvier 1987 à juillet 1998, nos 63-200)

76 Nous avons également trouvé quelques occurrences des termes «intermède» dans la presse francophone (dès I984) et d' «intermission» dans la presse anglophone (dès I982), bien que ces expressions soient largement minoritaires en regard des termes établis pour qualifier les séquences non interactives.

77 La variante orthographique "cutscene», sans tiret, a également été considérée dans ce décompte.

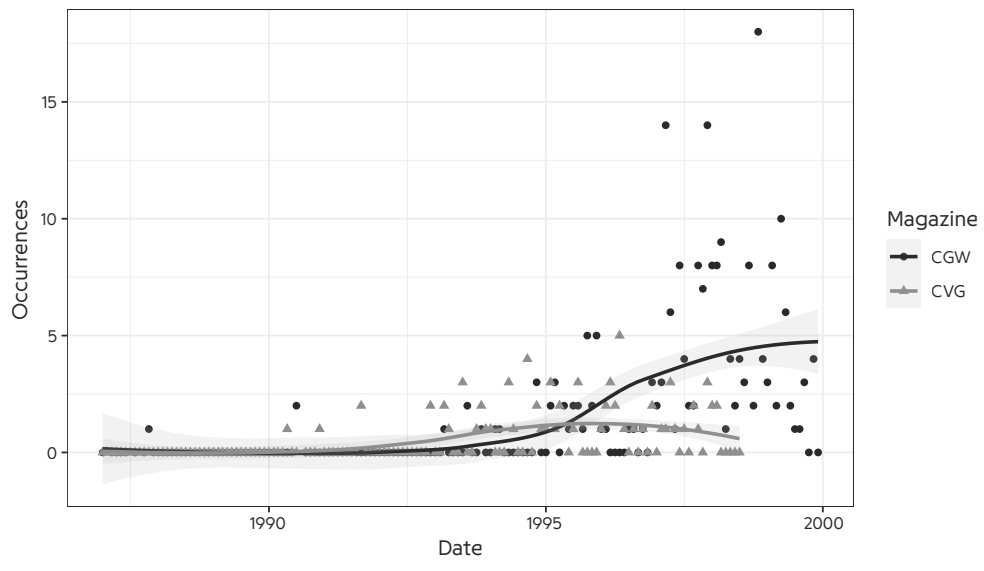

Notons par ailleurs que l'expression «séquence cinématique» est alors en concurrence avec le syntagme «séquence intermédiaire ${ }^{76}$ (ou «scène intermédiaire») qui est majoritairement employé, dans Tilt et Joystick, pour qualifier les segments non interactifs au début des années 1990. L'expression qui qualifie d'«intermédiaires» certaines «séquences», «scènes» ou encore «écrans» constitue probablement une traduction du vocable «cut-scene» qui vise à qualifier les séquences non interactives. Comme le rappelle Sébastien Genvo dans le présent dossier, le terme «cut-scene» apparaît d'abord dans le discours de production à l'occasion de la sortie du jeu d'aventure Maniac Mansion (i984) de LucasArt Games, alors que son créateur, Ron Gilbert, cherchait un terme pour qualifier les interludes qui ponctuent le jeu. L'analyse quantitative menée ici nous montre cependant qu'au sein des deux magazines anglophones traités, l'expression "cut-scene» ${ }^{77}$ se généralise plus tardivement, dans la seconde moitié des années 1990 ( fig. I2).

\section{La parenthèse Delphine}

Dans le magazine Joystick, on repère un premier «pic» d'apparition du terme de «cinématique» en I990. Ces occurrences sont d'autant plus marquantes qu'elles attestent l'usage de la forme substantive du terme «cinématique» (soit «la» cinématique). Cependant, l'ensemble de ces occurrences découle du choix de l'éditeur français Delphine 
qui nomme "Cinématique» la série de jeux d'aventure initiée par le jeu Les Voyageurs du Temps en i989. Les six occurrences du terme de «cinématique» dans le numéro inaugural du magazine français renvoient ainsi à la série éponyme du studio Delphine ${ }^{78}$. Le terme apparaît également dans le numéro de décembre i990, cette fois-ci à l’occasion d'un article prévisionnel concernant la sortie prochaine du deuxième jeu de la série "Cinématique», Operation Stealth (Delphine Software, 1990):

«En ce qui concerne le mode de fonctionnement du jeu, c'est le trip habituel de Mr Delphine Software: la cinématique. Vous indiquez, grâce à la souris ou au clavier, ce que vous voulez que le personnage fasse. En pointant une porte, et en sélectionnant, grâce aux menus déroulants, l'option à actionner, la porte s'ouvrira, par exemple. Ce mode d'utilisation déjà utilisé pour Les voyageurs du temps (du même éditeur), a été amélioré ici. Il n'est plus utile d'être près d'un objet pour le sélectionner.» ${ }^{79}$

La cinématique est ainsi pensée comme un «modèle» de gameplay, une interface graphique caractéristique des productions Delphine qui se présente comme un aménagement des interfaces «points \& click» qui se généralisent dans les jeux d'aventure durant la deuxième moitié des années $1980^{80}$. La presse emprunte donc ici directement le terme au discours de production.

S’il faudra encore attendre de nombreuses années avant que le substantif féminin «cinématique» réapparaisse dans les discours, l'emploi privilégié par la compagnie Delphine aura cependant créé un précédent notable, certainement inspiré par l'emploi fréquent de l'adjectif «cinematic» dans le discours promotionnel anglophone dès la seconde moitié des années ig8o.

Dans la première moitié des années 1990, il était par ailleurs fréquent que les journalistes recourent au terme «cinématique» pour décrire les jeux Delphine. En décembre 1992, les quatre occurrences du terme «séquence cinématique» dans les pages de Joystick renvoient ainsi au jeu d'aventure et de plateforme Flashback de Delphine. En novembre de la même année, le magazine Tilt consacre deux pages au jeu à venir de Delphine, en indiquant que ce titre «profit $[\mathrm{e}]$ du savoir-faire de Delphine en matière de cinématique», avant de préciser entre parenthèses: «mise en scène proche de celle du cinéma, animation très réaliste ${ }^{81}$. Le
78 Il en va de même pour les occurrences du terme dans les $\mathrm{n}^{\circ} 7$ (juillet 1990), $\mathrm{n}^{\circ} 9$ (octobre 1990), ${ }^{\circ}$ Io (novembre 1990) et nº II (décembre 1990).

79 Joystick, $\mathrm{n}^{\circ}$ II, décembre I990, p. 226. Le terme apparaît également dans la presse anglophone à l'occasion de la réception des jeux d'aventure de Delphine. Un article de CGW évoque ainsi le «Cinematique System» à l'occasion de la sortie américaine de Croisière pour un $\mathrm{Ca}$ davre en le qualifiant d'«interface de jeu» [«playing interface»]. CGW $\mathrm{n}^{\circ}$ IоI, décembre 1992, p. 72.

80 Au sujet de la transition «cinématographique» du jeu d'aventure, nous renvoyons le lecteur à l'article de Sébastien Genvo dans ce numéro de Décadrages.

81 Tilt, $\mathrm{n}^{\circ}$ 107, novembre 1992 , p. I2: «Profitant du savoir-faire de Delphine en matière de cinématique (mise en scène proche de celle du cinéma, animation très réaliste), Flashback s'annonce comme un hit en puissance.» Précisons qu'il s'agit ici d'une occurrence, en légende d'une image, qui n'a pas été détectée par notre logiciel d'OCR. Elle a donc été relevée à la main de manière fortuite! 
82 Id., p. I3. Nous soulignons.

83 La boîte du jeu présente notamment les atouts suivants: "Stunning 24 frame per second screen updates resulting in superb animation»; "The smoothest, most lifelike characters ever seen»; «Compelling movie atmosphere». Matériel consulté sur www.mobygames.com. journaliste reconduit ensuite - avec enthousiasme - le discours promotionnel du studio Delphine au sujet de son plateformer:
«[...] Delphine nous promet une réalisation extraordinaire. Les mou- vements, représentés par 24 images/seconde (contre 7 pour le dessin animé moyen), s'inspirent de films avec de vrais acteurs. Le système Rotoscoping (semblable à celui d'Another World) livre des personnes et des animations très réalistes. Comme d'habitude, Delphine aug- mentera le suspens à l'aide de séquences intermédiaires cinématiques, de zooms, close up et perspectives dramatiques. Plus qu'un jump'n'run [jeu de plateforme] raffiné, Flashback promet de devenir une aventure à vous couper le souffle. Nous attendons impatiemment la version finale...» 82

La volonté de «faire cinéma», caractéristique des productions de Delphine dès la fin des années i980, transparaît très nettement dans le discours du journaliste qui évoque, sous la forme d'un inventaire, les différentes «innovations» propres au jeu en question. Notons que les articles prévisionnels - présentés dans la rubrique «avant-premières» de Tilt qui emprunte elle-même au lexique cinématographique - font habituellement suite à une prise de contact entre les éditeurs et l'équipe de rédaction. Les éditeurs (ou les diffuseurs) confient alors aux journalistes le matériel promotionnel du jeu ainsi qu’une version «démo» qui leur permet de couvrir, en exclusivité, un jeu vidéo donné. Ces «articles» sont donc particulièrement susceptibles de reproduire (parfois mot pour mot) le discours promotionnel de l'instance de production. A ce titre, la description des atouts d'un logiciel, présentés sous la forme d'un inventaire, apparaît très fréquemment dans les publicités ou les boîtes des jeux vidéo, offrant un listing des attraits et des innovations du jeu au client potentiel.

L'édition européenne de Flashback pour Amiga (I992) énumère sur le verso de sa boîte sept «qualités» (présentées comme des «faits»), parmi lesquelles on retrouve le «rafraîchissement de l'écran en 24 images par seconde», la qualité «réaliste» de l'animation ou encore «l'atmosphère cinématographique envoûtante ${ }^{83}$, témoignant ainsi de la proximité entre le discours promotionnel de la production et le discours de presse.

Outre la modalisation forte du discours et l'emploi de termes empruntés au lexique cinématographique, on remarque que le journaliste 
croise ici les deux expressions alors en vigueur pour qualifier les séquences non interactives lorsqu’il parle de «séquences intermédiaires cinématiques», montrant ainsi que l'emploi de ces termes est encore instable au début des années 1990 (avant que le terme de «séquence intermédiaire» ne disparaisse progressivement des usages) ${ }^{84}$.

\section{Substantivation du terme}

Si les productions Delphine suscitent l'utilisation du terme «cinématique» au sein de la presse francophone, il ne faut cependant pas surestimer leur rôle dans le processus de lexicalisation de cette notion. Effectivement, dès 1992, le terme vient qualifier une grande diversité de productions vidéoludiques. L'emploi de cette notion sera alors favorisé par le «tournant cinématographique» qui marque l'industrie du jeu vidéo dans les années I99o et qui dépend d’un faisceau pluriel de déterminations, à la fois économiques, techniques et culturelles. La généralisation de la 3D polygonale, l'utilisation accrue de textures photographiques ainsi que l'ajout de séquences non interactives en images de synthèse précalculées touchent ainsi une grande quantité de productions et de genres durant cette décennie.

En juillet I993, dans le magazine Tilt, le syntagme «séquence [ou scène] cinématique» apparaît à six reprises, pour qualifier des segments de jeux d'aventure (The Dig, LucasArts, 1995; Kyrandia 2, Westwood Studios, 1993), de simulations de combat spatial (Rebel Assault et $X$-Wing, I993), et d'un jeu de stratégie (Stronghold, Stormfront Studios, 1993) ${ }^{85}$. Alors que le terme se généralise dans la deuxième moitié des années I990, il est employé pour qualifier une grande diversité de jeux, bien au-delà des seuls jeux d'aventure graphique à laquelle son utilisation était liée durant la seconde moitié des années i980. En guise d'exemple, mentionnons le numéro 72 de la revue Joystick (juin i996) qui accueille i6 occurrences de l'expression «séquence/scène cinématique» pour qualifier des séquences de jeux d'aventure, de jeux de stratégie, de jeux de sport, de simulations de course automobile, de films interactifs, de jeux d'épouvante, ou encore de simulateurs de vol! ${ }^{86}$ C'est alors dans le contexte d'une lexicalisation progressive des expressions «scène cinématique» et «séquence cinématique» qu’un processus de substantivation s'amorce progressivement en français à partir de 1996.
84 On trouve un autre exemple dans le magazine Joystick, lorsqu'un journaliste qualifie les séquences du film Top Gun intégrées dans son adaptation vidéoludique de «scènes cinématiques intermédiaires ». Joystick, mars I996, n 69, p. 97.

85 Tilt, n ${ }^{\circ}$ Ir6, juillet I993, pp. 26-27. On retrouve ici deux adaptations issues de la franchise Star Wars ainsi qu'un jeu d'aventure dont l'«idée originale» est attribuée à Steven Spielberg, qui se sera lui-même intéressé à l'industrie du jeu vidéo dans les années I990. La mention relative à $X$-Wing apparaît dans une publicité pour l'extension Imperial Pursuit qui indique que cette dernière est pourvue «de nouvelles séquences cinématiques avec musique et effets sonores».

86 Joystick, $\mathrm{n}^{\circ}$ 72, juin 1996. «Séquence [ou scène] cinématique» (singulier ou pluriel) est employé dans la présentation des jeux Down in the Dumps (Haiku Studio, 1996), Secret Mission (portage CD-i d'Aventure de Microids, I996), Normality (jeu d'aventure du studio Gremlins, I996), Dark Earth (jeu de Kalisto Entertainment inspiré de Alone in the Dark, I997), Olympic Games (US Gold, I996), Urban Runner (film interactif de Coktel Vision, I996), Wing Commander IV (Origin Systems, I996), MegaRace 2 (Cryo Interactive Entertainement, I996), Normality (Gremlin Interactive, I996) ou encore l'extension de Warcraft II (Blizzard Entertainment, 1995). Les Chevaliers de Baphomet (Revolution Software, I996) est par ailleurs qualifié d'«aventure cinématique» (p. 56 ). 
87 Joystick, $\mathrm{n}^{\circ} 73$, juillet i996, p. го. A noter que la boîte du jeu qualifie les segments non interactifs de «séquence d'animation générée en $3 \mathrm{D}$ » [3-D rendered animation] et n'utilise pas le terme de «cinématique».

88 Joystick, n 75, octobre i996, pp. 8283. Ce retour sur le jeu de 1993 se justifie par la sortie prochaine du dernier jeu de Tribolyte, Clandestiny. Le journaliste parle également de «belles cinématiques».

89 Joystick, $\mathrm{n}^{\circ} 76$, novembre i996, p. Io6. 90 On compte, par exemple, trois occurrences du substantif pluriel «cinématiques» dans le numéro de Joystick de juin 1997 (nº3).

13/ Les occurrences du terme "cinematics» dans Computer Gaming World (de janvier 1992 à décembre 1999, nº $90-185)$
En juillet I996, un critique de Joystick évoque ainsi les «superbes cinématiques» du jeu de stratégie Lords of the Realm (Impressions Games, I994) ${ }^{87}$. En octobre I996, un critique revient sur l'engouement suscité par The $7^{\text {th }}$ Guest (Trilobyte, I993) en invoquant, comme principal facteur de son succès, «ses nombreuses cinématiques» ${ }^{88}$. Un mois plus tard, c'est au tour de Deadly Tide de Microprose d'être discuté à l'aune de ses nombreuses "cinématiques» ${ }^{89}$. Si le substantif apparaît alors moins fréquemment que l'adjectif «cinématique», il gagne cependant du terrain et se répand considérablement à partir de l'été $1997^{90}$. En 1998 - dernière année traitée dans cette étude - le substantif "cinématique» apparaît encore au côté des syntagmes figés «scène cinématique»et «séquence cinématique». Ce processus de substantivation se poursuit ensuite dans les années 2000 pour conduire à la naturalisation du terme de «cinématique».

\section{La cinématique: une invention anglophone?}

La substantivation du terme apparaît également dans la presse anglophone, où elle se produit quelques années plus tôt. Une fois de plus, le discours de production joue un rôle important dans la lexicalisation du terme. La première occurrence que nous avons repérée dans le corpus anglophone apparaît au sein d'une publicité pour le jeu de rôle The Summoning (SSI, I992) qui vante la présence de «cinématiques» au sein du

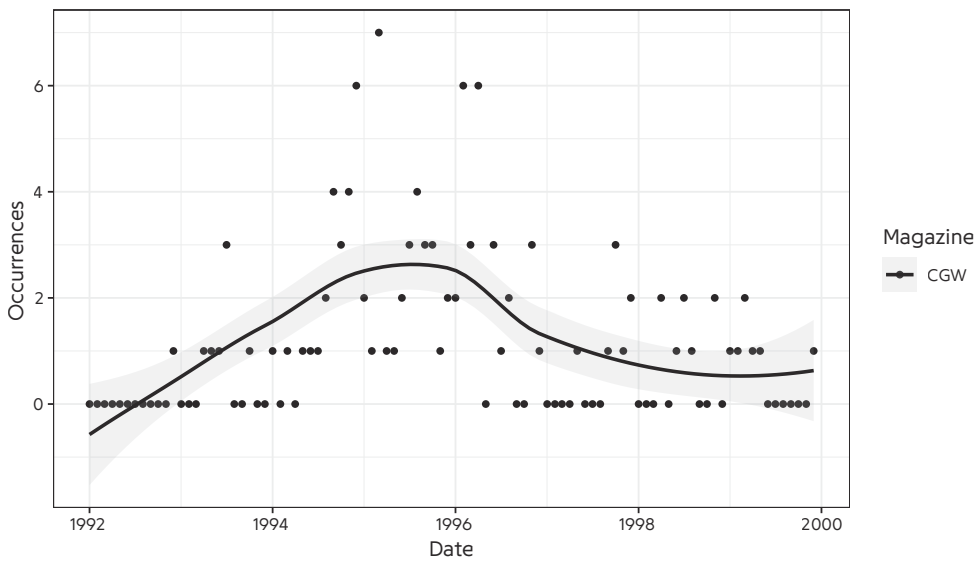


jeu ${ }^{91}$. A partir de i994, le substantif pluriel «les cinématiques» [«cinematics»] apparaît fréquemment dans le magazine américain CGW, constituant ainsi une alternative au vocable «cut-scene» qui se répand également (fig. 13). En décembre 1994, le substantif pluriel apparaît à six reprises dans les pages de $C G W$, alors qu'à la même époque la presse française n’a pas encore conduit l'élision des termes «séquence» et «scène».

Parmi les six occurrences, quatre proviennent de réclames pour des jeux de rôle ou des simulations spatiales. On remarque alors que certains éditeurs américains, à l'image de SSI, intègrent le substantif «cinématique» à leur discours promotionnel et le servent alors «à toutes les sauces». L'ensemble des jeux SSI promus dans ce numéro vantent ainsi l'innovation induite par la généralisation des supports optiques et font des «graphismes», mais aussi des «cinématiques et des voix, musiques et bruitages numérisés» des arguments de vente décisifs de leurs productions ${ }^{92}$. Le terme apparaît également dans le discours promotionnel des productions du studio Origin et de sa série Wing Commander. En i994, la version «Sega-CD» de Wing Commander se vante «d'inclure dans un seul et unique disque optique des cinématiques ainsi que des séquences vocales numérisées ${ }^{93}$. Il semblerait ainsi que la lexicalisation du terme en anglais accompagne l'arrivée des supports optiques sur le marché et constitue une «innovation lexicale» qui vient répondre à l'injonction d'innovation technique constitutive de l'industrie du jeu vidéo, marquée par des logiques complémentaires de «dépassement et d'obsolescence» ${ }^{94}$.

Le processus de lexicalisation du terme «cinématique» découle donc d'un anglicisme au sein du paratexte vidéoludique qui conduit à la transposition de l'adjectif «cinematic» en «cinématique» au début des années 1990. Le terme qualifie majoritairement, à partir de 1992-I993, les substantifs «séquence», «scène» ou «écran» afin de désigner des segments vidéoludiques non interactifs. Ces expressions, qui contiennent la forme adjectivale du terme "cinématique», céderont progressivement le pas à «la cinématique», dès I996-I997. Ce processus de substantivation se produit d'abord en anglais aux alentours de 1994, au moment de la diffusion massive des supports de stockage optiques, où il est initié par certains acteurs de la sphère de production dans le domaine des simulations de combat spatial et des jeux de rôle (SSI, Sir-Tech, Origin), pour être ensuite reconduit dans le discours de presse.
91 «Relish the splendidly detailed graphics, cinematics, sound effects and musical score.» (CGW, $\mathrm{n}^{\circ}$ IoI, décembre 1992 , p. II3). Nous soulignons.

92 Voir les réclames pour les jeux de l'éditeur SSI CyClones (jeu de tir) et ses «incroyable cinématiques» (p.92), Dark Sun (jeu de rôle de la licence AD\&D) et ses "cinématiques spectaculaires» (p. II5) et Renegade: Battle for Jakob's Star qui jouit de «Super-VGA graphics, cinematics, digitized voices, music and sound effects» (p. 327). Le substantif pluriel «cinématiques» apparaît également dans une publicité pour le jeu de rôle Star Trail (Sir-Tech, p. I84). Les journalistes parlent quant à eux des "cinématiques» de Wing Commander III (p.52) ainsi que des «cinématique en $3 \mathrm{D}$ » du jeu Battle Isle 2200 ( $3-\mathrm{D}$ cinematics and changing camera views", p. 72).

93 Boîte de Wing Commander, version Sega-CD, USA, I994. Consulté sur www.mobygames.com.

94 Voir James Newman, Best Before: Videogames, Supersession and Obsolescence, New York, Routledge, 2012. 
Les outils informatiques de récolte et de traitement de données textuelles que nous avons employés dans le cadre de ce projet auront considérablement facilité l'obtention des résultats présentés dans cet article et nous auront permis de baliser l'émergence et la généralisation du terme de «cinématique» dans les discours de presse. Nous avons pu relever le rôle fondamental du discours promotionnel dans le processus de «fabrication» de cette notion, qui reflète les ambitions cinématographiques de nombreux acteurs de l'industrie vidéoludique dès la fin des années i980 et qui permet d’infléchir le «cadrage» des jeux vidéo qu’elle désigne.

Le terme de «cinématique», tout en désignant des segments vidéoludiques qui empruntent aux codes et aux conventions du cinéma, constitue par ailleurs une «notion» dont l'histoire nous renseigne sur l'évolution des schèmes de perception qui se sont constitués au sein des communautés du jeu vidéo et qui, aujourd'hui encore, convoquent régulièrement le cinéma ainsi que les termes qui lui sont associés. 